\title{
IMMUNOHISTOCHEMICAL FEATURES OF GASTROINTESTINAL STROMAL TUMORS AND THEIR ROLE FOR DIFFERENTIAL DIAGNOSIS AND PROGNOSIS
}

\author{
Yana Miroshnichenko \\ Department of Pathological Anatomy \\ Kharkiv Medical Academy of Postgraduate Education \\ 58 Amosova str., Kharkiv, Ukraine, 61176 \\ pathologist.yana@gmail.com
}

\begin{abstract}
The aim. To clarify all most important immunohistochemical features of gastrointestinal stromal tumors with different histological patterns and analyze the role of expression of Ki-67, MMP-9, VEGF and p16ink4A as a predictive markers of tumor progression.

Materials and methods. The study is based on analysis of 100 primary GISTs for description of their morphological features and 36 GISTs taken from this 100 for study of prognostic markers.

Results. All spindle cell GISTs have shown diffuse expression of CD117 in tumor cells. The levels of CD117 expression varied from strong expression (3+) until mild expression (1+). Strong expression were seen in $75.8 \%$ of spindle cell GISTs. Epithelioid GISTs demonstrated heterognous moderate or mild expression of CD117. All primary epithelioid GISTs from patients that had relapse of tumor in period from 1 till 3 years demonstrated focal mild expression of CD 117 in tumor cells. Expression of DOG-1 were seen in all 100 cases of GISTs, that were included in our study. The strong expression of DOG-1 (3+) were seen in all 45 GISTs that had low mitotic rate ( $\leq 5$ mitoses per $50 \mathrm{HPF})$ and not associated with their histological pattern. GISTs with high mitotic rate demonstrated heterogeneous expression of DOG-1 in tumors: moderate expression (2+) with patchy areas of strong expression $(3+)$. Expression of CD56 was not found in spindle cell GISTs, but single tumor cells of epithelioid GISTs that had high mitotic rate demonstrated expression of this marker. The average expression of p16ink4A were higher in tumors that gave relapses compared with tumors without relapses $(50.3 \%$ versus $5.7 \%$ respectively, U-test $=16.5 ; p \leq 0.01)$. The average expression of MMP-9 also were significantly higher in GISTs that gave relapses: $63.2 \%$ compared with $13.4 \%$ in GISTs without relapse (U-test $=16 ; p \leq 0.01$ ). The strong VEGF expression was found in $66.7 \%$ of GISTs that had relapses and only in $8.3 \%$ of GISTs without relapses. $50 \%$ of GISTs without relapses was negative for VEGF. Finally, the average expression of Ki-67 were $13.4 \%$ in GISTs with relapses and $8.7 \%$ in GISTs without them $(\mathrm{U}$-test $=16 ; p \leq 0.01)$.

Conclusion. We highly recommend using DOG-1 for epithelioid GISTs. Additionally in epithelioid GISTs can be used CD56 that can give focal positive reaction in some tumour cells. The following minimal panel of markers for differential diagnosis of spindled GISTs from other mesenchymal tumors of gastrointestinal tract is proposed: CD117, DOG-1 and SMA, where the first too markers will demonstrated the moderate or strong diffuse expression and SMA can be occasionally positive in some tumor cells. p16ink4A, Ki-67, VEGF and MMP-9 can be used as additional prognostic markers in GISTs.
\end{abstract}

Keywords: CD117, DOG-1, CD56, spindled GISTs, epithelioid GISTs, prognostic markers, p16ink4A, Ki-67.

DOI: $10.21303 / 2504-5679.2021 .002183$

\section{Introduction}

Gastrointestinal stromal tumors (GIST) are the most common mesenchymal tumors of digestive system [1,2]. The correct determination of these tumors are crucial for choosing the best strategy of treatment and prediction of tumor behavior. GISTs can share 2 most common histological patterns: spindle cell morphology and epithelioid type. Nowadays the diagnosis GIST is not be full without immunohistochemical (IHC) testing with CD117 and/or DOG-1 [3, 4]. The spindled GISTs should be differentiated from leiomyomas and schwannomas. Epithelioid GISTs are required differential diagnosis with huge specter of round cell tumors, starting from carcinoids and finishing with glomus tumors of stomach [5,6]. GISTs have variable behavior; many of them are characterized by indolent course. However, at the same time, there are too less histological criteria, that allow to predict progression of GISTs. Criteria that were used in last histological classification of digestive system tumors include mitotic rate, tumor size and tumor site of location and only 
$1^{\text {st }}$ criterion in this list is histological $[1,2,7]$. The IHC marker, such as widely used in different type of malignancies for analysis of their aggressiveness can be also used in GISTs as additional markers for prognosis of tumor behavior. The most popular markers from this group are Ki-67 for evaluation of proliferative activity [8], MMP-9 that helps to check the ability of a tumor to invasion [9] and VEGF - the marker of angiogenesis [10]. P16ink4a is a particularly potent effector of cell cycle progression that functions in concert with CDK4/Cyclin D and RB in coordinating proliferation. Application of immunohistochemical analysis with pl6ink4A can be used as a novel biomarker for the detection of cancer cells in early stages [11].

The scientific papers that give complex study of immunohistochemical features of GISTs and analyze the markers of tumor aggressive behavior in groups with and without relapses are almost absent or they describe only couples of markers.

The aim of this study was to clarify all most important immunohistochemical features of gastrointestinal stromal tumors with different histological patterns and analyze the role of expression of Ki-67, MMP-9, VEGF and p16ink4A as a predictive markers of tumor progression.

\section{Material and methods}

The study was performed on formalin fixed and paraffin embedded (FFPE) tumor samples of GISTs. The material included tumor samples of primary GISTs obtained from 100 patients, who have undergone surgical excision of tumors. Postoperative tumor material was obtained from pathology departments of «Grigoriev Institute for Medical Radiology and Oncology of the National Academy of Medical Sciences of Ukraine», «V. T. Zaycev Institute of General and Urgent Surgery of NAMS of Ukraine» Kharkiv, Ukraine and «National Cancer Institute», Kyiv, Ukraine. All surgical resections were performed between 2013 and 2019.

The design of the study and all the methods used in the study were approved by the Bioethics Committee of the Kharkiv medical academy of postgraduate education, protocol 9 (21.11.2018) and complied with the requirements of the Declaration of Helsinki. Such clinic-morphological criteria as gender, site of tumor and its size were obtained from case histories, where we analyzed surgical protocols, data about chemotherapy and data from histological conclusions.

Eligibility criteria included the availability of follow-up data at least for a year after surgical resection of primary tumor positive immunohistochemical staining with CD117 and DOG-1 confirming diagnosis GIST, presence of information about staining with SMA or available blocks with tumors with sufficient material for at least 4 histological sections. Additionally, we made IHC testing with CD34, CD56, SMA, DOG-1 if it was not performed previously. The information of 100 cases of GISTs is given in Table 1.

Table 1

Clinico-morphological characteristic of primary GISTs

\begin{tabular}{|c|c|c|c|}
\hline \multicolumn{4}{|c|}{$\operatorname{Sex}(\mathbf{N})$} \\
\hline Males & & Females & \\
\hline 35 & & 65 & \\
\hline \multicolumn{4}{|c|}{ Age (mean) } \\
\hline \multicolumn{4}{|c|}{$57.96 \pm 8.2$} \\
\hline \multicolumn{4}{|c|}{ Histological pattern $(N)$} \\
\hline Spindled GISTs & Epithelioid GISTs & & Mixed \\
\hline 91 & 3 & & 3 \\
\hline \multicolumn{4}{|c|}{ Mitotic activity } \\
\hline$\leq 5 / 50 \mathrm{HPF}$ & & $>5 / 50 \mathrm{HPF}$ & \\
\hline 45 & & 5 & \\
\hline \multicolumn{4}{|c|}{ Relapse in period from 1 till 3 years after primary surgery } \\
\hline Yes & & No & \\
\hline 37 & & 63 & \\
\hline
\end{tabular}


For ancillary study with markers of tumor aggressive behaviour (Ki-67, p16ink4A, VEGF and MMP-9) we choose 36 GISTs from 100 cases that have enough amount and good quality of material in formalin fixed and paraffin embedded (FFPE) tissue samples. Among 36 GISTs that we have chosen 12 had relapses in period from 1 till 3 years and 24 patients were without relapses in the same time lap. The characteristics of antibodies are given in Table 2 . The whole study was performed according to the Dako protocol for manual IHC staining.

Table 2

List of primary antibodies used in our study

\begin{tabular}{cccc}
\hline Primary antibody & Clone & Dilution & Manufacturer \\
\hline DOG-1 & SP31 & $1: 100$ & ThermoFisher Scientific, USA \\
CD117 & A4502 & $1: 250$ & Dako, Denmark \\
SMA & $1 \mathrm{~A} 4$ & $1: 300$ & ThermoFisher Scientific, USA \\
CD34 & QBEnd/10 & $1: 500$ & ThermoFisher Scientific, USA \\
CD56 & $123 C 3$ & $1: 100$ & \\
MMP-9 & Ab-1 GE-213 & $1: 200$ & ThermoFisher Scientific, USA \\
Ki-67 & SP6 & $1: 400$ & ThermoFisher Scientific, USA \\
VEGF & JH121 & $1: 20$ & ThermoFisher Scientific, USA \\
P16ink4A & 1D7D2 & $1: 200$ & ThermoFisher Scientific, USA
\end{tabular}

Quantitative method was used for evaluation of Ki-67, MMP-9 and p16ink4A expression.

Expression of p16ink4A was calculated as the percentage of the number of immunopositive cells (positive cytoplasmic staining with or without nuclear staining) among the total number of tumour cells in three areas of most intensive staining (in cases of heterogeneous expression) at $\times 400$ magnification.

The semiquantitative approach was used for the assessment of VEGF, DOG-1, CD117, SMA, CD34 and CD56 and expression.

Results were visualized and photographed using light microscope (ZEISS Primo Star, ZEISS Axiocam ERc5).

The Mann-Whitney U test was used to compare differences between expression of markers in group of GISTs with relapses in period from 1 till 3 years after surgery and without them in this time lap (P-value < 0.01). An extensive parameter (\%) was used to describe qualitative characteristics. All statistical analyses were performed using «Microsoft Excel 2013» and «MedCalc».

\section{Results}

All spindle cell GISTs have shown diffuse expression of CD117 in tumour cells. The levels of CD117 expression varied from strong expression (3+) until mild expression (1+). Strong expression were seen in $75.8 \%$ of spindle cell GISTs (Fig. 1).

Mild expression of CD 117 in our study were more typical for tumors with mitotic rate higher that 5 mitoses per 50HPF (among 9 tumors with mild CD117 expression, 6 tumors had mitotic rate higher that 5 mitoses per 50HPF). Moreover, tumors with mild expression of CD117 had usually stroma with areas of mixomatosis and small necrosis. Epithelioid GISTs demonstrated heterognous moderate or mild expression of CD117. All primary epithelioid GISTs from patients that had relapse of tumor in period from 1 till 3 years demonstrated focal mild expression of CD 117 in tumor cells (Fig. 2).

Expression of DOG-1 were seen in all 100 cases of GISTs, that were included in our study. The strong expression of DOG-1 $(3+)$ were seen in all 45 GISTs that had low mitotic rate $(\leq 5 \mathrm{mi}-$ toses per 50HPF) and not associated with their histological pattern. GISTs with high mitotic rate demonstrated heterogenous expression of DOG-1 in tumors: moderate expression $(2+)$ with patchy areas of strong expression (3+).

All epithelioid GISTs in our study were negative for CD34. The spindle cell GISTs have shown next levels of CD34 expression: $35.2 \%$ (32 samples) of tumors had strong expression of CD34, $32.9 \%$ (30 samples) - moderate expression and $31.9 \%$ (29 samples) negative expression in tumor cells. 


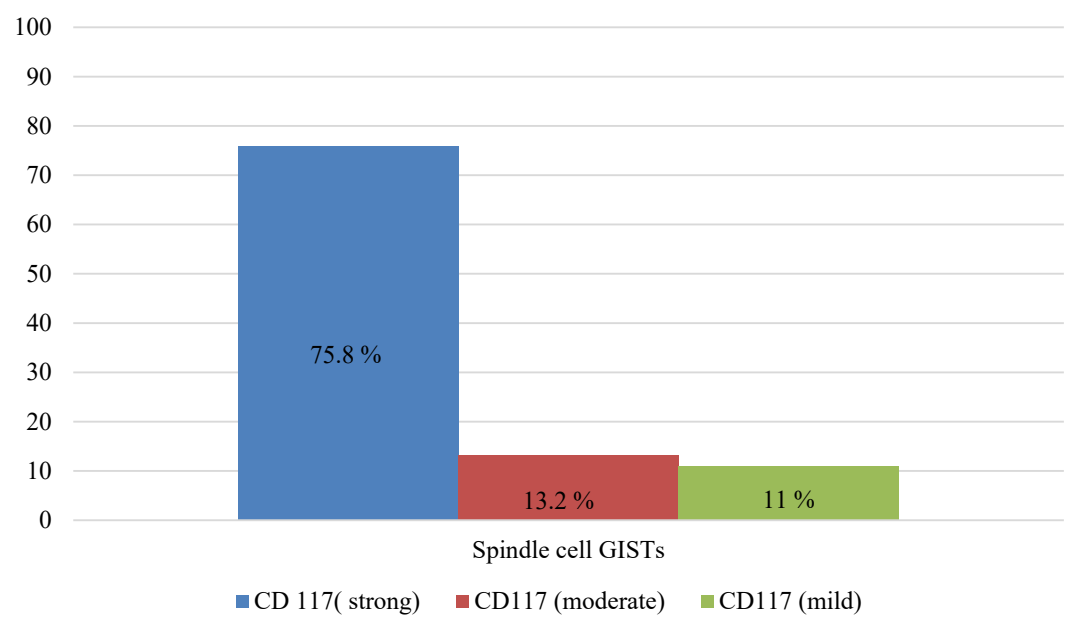

Fig. 1. Expression of CD117 in spindle cell GISTs

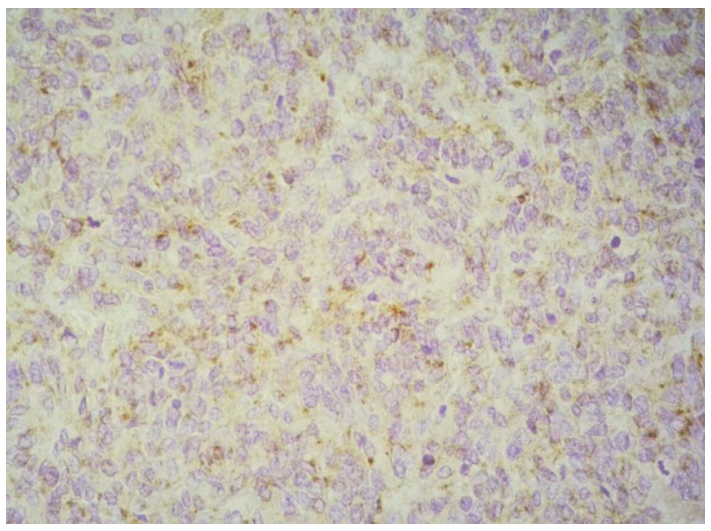

Fig. 2. Mild to moderate focal expression of CD 117 in epithelioid GIST, $\times 1000$

Expression of SMA was not detected in epithelioid GISTs, but in spindle cell GISTs occasional expression of SMA in single tumor cells were seen in $13.2 \%$ of cases.

Expression of CD56 was not found in spindle cell GISTs, but single tumor cells of epithelioid GISTs that had high mitotic rate demonstrated expression of this marker (Fig. 3).

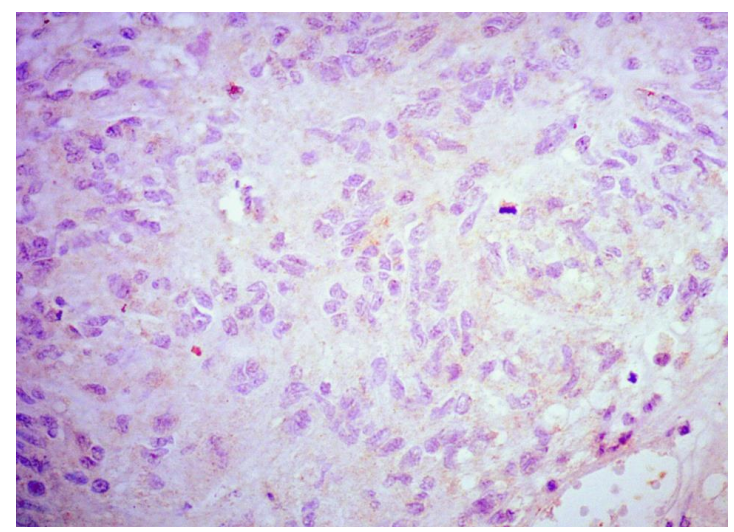

Fig. 3. Expression of CD 56 in single tumor cells of epithelioid GIST, $\times 1000$

Among 100 primary GISTs in our study we have only 3 tumors with mixed histological pattern. All these tumors have shown moderate expression of CD117 and strong expression of DOG-1.

The analysis of expression of IHC markers of tumor aggressive behavior such as p16ink4A, MMP-9, VEGF and ki-67 were performed on 36 cases of GISTs that were taken from our primary 
100 cases. The relapse of tumor in period from 1 till 3 years were seen in $33.3 \%$ of cases (12 patients) and other $66.7 \%$ of cases did not develop relapse in mentioned time lap.

The average expression of p16ink4A were higher in tumors that gave relapses compared with tumors without relapses $(50.3 \%$ versus $5.7 \%$ respectively, $\mathrm{U}$-test $=16.5 ; p \leq 0.01)$.

The average expression of MMP-9 also were significantly higher in GISTs that gave relapses: $63.2 \%$ compared with $13.4 \%$ in GISTs without relapse (U-test $=16 ; p \leq 0.01$ ).

The strong VEGF expression was found in $66.7 \%$ of GISTs that had relapses and only in $8.3 \%$ of GISTs without relapses. $50 \%$ of GISTs without relapses was negative for VEGF (Fig. 4).

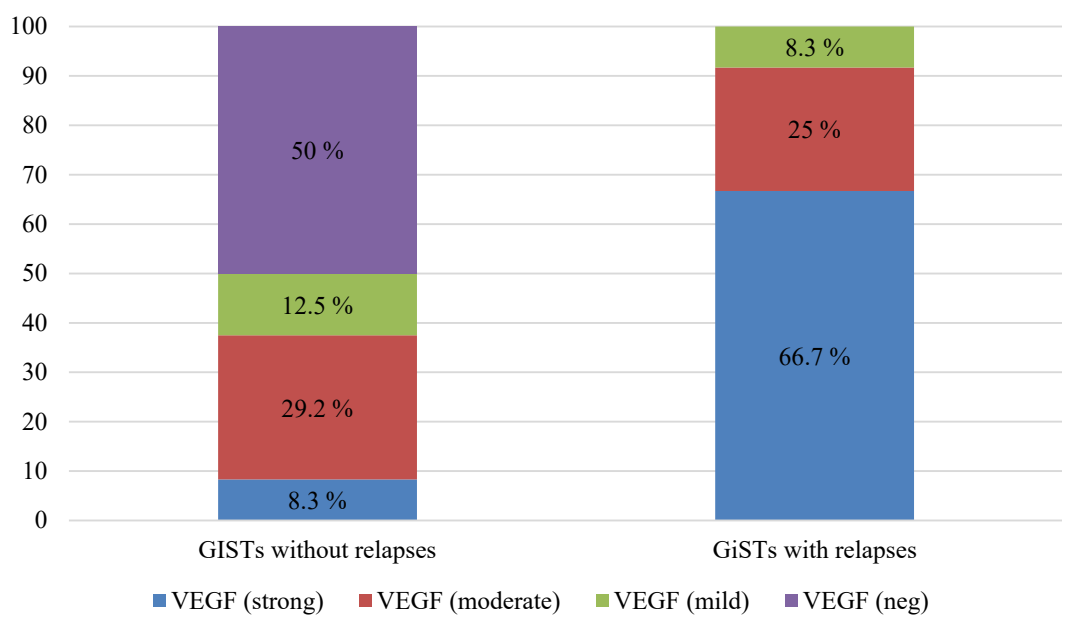

Fig. 4. Expression of VEGF in GISTs with and without relapses in period from 1 till 3 years

Finally, the average expression of Ki-67 were $13.4 \%$ in GISTs with relapses and $8.7 \%$ in GISTs without them (U-test $=16 ; p \leq 0.01)$.

\section{Discussion}

The data that we obtained in our study show that analysis of CD 117, DOG-1 and SMA expression is important for differential diagnosis of spindle cell GISTs. The first 2 markers are also recommended in protocol for GIST examination given by College of American pathology [12]. The analysis of SMA expression is important for differential diagnosis between spindle cell GISTs and leiomyomas $[1,5,12]$. Although numerous data talk about absence of SMA expression in GIST [1], some authors reported about occasional expression of SMA in some tumour cells in GISTs [13, 14]. Such an occasional expression in a few tumour cells we have seen in our study too. In our study, we also described focal expression of CD56 in epithelioid GISTs. The similar finding was reported in study of A. Agaymy and P. H. Wünsch, who said that focal expression of CD56 can be seen in $50 \%$ of epithelioid and only $7 \%$ of spindled GISTs [15]. Epithelioid GISTs in our study demonstrated weaker expression of CD117 compared with spindled GISTs, some other authors also said about such weak focal expression of CD117 in epithelioid gastric GISTs and mentioned that such group of tumours have PGFRA mutations [16, 17]. Especially for such subtype of tumors is recommended to perform IHC analysis with DOG-1, that is more sensitive for GISTs [12, 18]. In our study we saw that tumors with mild heterogenous CD117 expression demonstrated moderate or strong expression of DOG-1. Expression of CD34 was negative in epithelioid GISTs. This data is very similar to data given by WHO in last classification of digestive system tumors $[1,7,19]$. The CD34 expression in spindled GISTs was seen in $68.1 \%$ of spindled GISTs in our study that not contradicted to different scientific data where this marker expressed approximately in $70 \%$ of GISTs $[12,13]$.

Analysis of expression of such markers as p16ink4A, ki-67, VEGF and MMP-9 demonstrated that expression of these markers were higher in GISTs that have relapses compared with GISTs without them. The expression of last 3 markers is widely described in different tumours and they are known as markers of aggressive behaviour. Different authors have shown that high expression of MMP-9, VEGF and Ki-67 can be a marker of poor prognosis in GISTs [8-10]. 
The high-level expression of p16ink4a in tumors is associated with aggressive subtypes of disease, and in certain clinical settings elevated p16ink4a expression is an important determinant for disease prognosis and therapeutic response [11, 20, 21]. F. Haller et al. in their article described the cytoplasmic expression of pl6ink4A as an independent factor of worse prognosis in GISTs [20]. All this data supports the hypothesis that these markers can be used as a prognostic marker in GISTs too. But ancillary study of these markers and influence of other factors of GISTs progression are required.

Study limitations. The study of prognostic markers requires analysis of larger groups of GISTs to find cut-off levels of markers expression that will be helpful for given a more accurate prognosis.

Prospects for further research. The further study of different molecular-biological features of GISTs will allow us to predict better behavior of these tumors and choose the best strategy of treatment for each patient.

\section{Conclusions}

We recommend using the following minimal panel of markers for differential diagnosis of spindled GISTs from other mesenchymal tumours of gastrointestinal tract: CD117, DOG-1 and SMA, where the first markers will demonstrate the moderate or strong diffuse expression and SMA can be occasionally positive in some tumour cells.

The use of DOG-1 is crucial for differential diagnosis of epithelioid GISTs, because this group of tumours can demonstrate only weak focal expression of CD117 in some tumour cells. Additionally, in epithelioid GISTs can be used CD56 that can give focal positive reaction in some tumour cells.

Such markers as p16ink4A, Ki-67, VEGF and MMP-9 can be used as additional prognostic markers in GISTs.

\section{Conflicts of interest}

The authors declare that they have no conflicts of interest.

\section{Funding}

This study was made as a part of research work: «Pathohistological and immunohistochemical diagnostics and prognosis of malignant tumours of different localisations based on their biological features and clinical course», state registration No. $0117 \mathrm{U} 000594$.

\section{Acknowledgments}

The authors thank the Department of Pathology of «Grigoriev Institute for Medical Radiology and Oncology of the National Academy of Medical Sciences of Ukraine», Kharkiv, Ukraine and «National Cancer Institute», Kyiv, Ukraine for providing post-operative human gastrointestinal stromal tumors tissue samples. We are also grateful to the Archives of these hospitals for provided access to patients' case histories and follow-up data about their treatment.

\section{References}

[1] Lokuhetty, D. (2019). Gastrointestinal stromal tumour. WHO Classification of Tumours: Digestive System Tumours. Lyon, 439-443.

[2] SEER Inquiry System - View (2011). National Cancer Institute: Surveillance, Epidemiology, and End Result Program. Available at: https://seer.cancer.gov/seerinquiry/index.php?page=view\&id=20100014\&type=q

[3] Gupta, A., Suri, V., Goyal, N., Chhabra, S., Garg, A., Bhati, G., Kaur, J. (2020). Histomorphological spectrum of gastrointestinal stromal tumors - An institutional experience in a series of seven cases. Adesh University Journal of Medical Sciences \& Research, 2, 112-116. doi: http://doi.org/10.25259/aujmsr_33_2020

[4] Ceausu, M., Socea, B., Ciobotaru, V., Constantin, V., Enache, S., Enache, V. et. al. (2021). A multidisciplinary approach in the diagnostic challenge of GIST. Experimental and Therapeutic Medicine, 22 (4). doi: http://doi.org/10.3892/etm.2021.10497

[5] Hirota, S. (2018). Differential diagnosis of gastrointestinal stromal tumor by histopathology and immunohistochemistry. Translational Gastroenterology and Hepatology, 3, 27-27. doi: http://doi.org/10.21037/tgh.2018.04.01 
[6] Gastrointestinal Stromal Tumor (GIST). Differential Diagnosis. Surgical Pathology Criteria. Stanford University School of Medicine. Available at: http://surgpathcriteria.stanford.edu/gitumors/gist-gastrointestinal-stromal-tumor/differential-diagnosis.html

[7] Gheorghe, G., Bacalbasa, N., Ceobanu, G., Ilie, M., Enache, V., Constantinescu, G. et. al. (2021). Gastrointestinal Stromal Tumors - A Mini Review. Journal of Personalized Medicine, 11 (8), 694. doi: http://doi.org/10.3390/jpm11080694

[8] Zhao, W. Y., Xu, J., Wang, M., Zhang, Z. Z., Tu, L., Wang, C. J. et. al. (2014). Prognostic value of Ki67 index in gastrointestinal stromal tumors. International journal of clinical and experimental pathology, 7 (5), 2298-2304.

[9] Xu, D., Su, C., Guo, L., Yan, H., Wang, S., Yuan, C. et. al. 2019). Predictive significance of serum MMP-9 in papillary thyroid carcinoma. Open Life Sciences, 14 (1), 275-287. doi: http://doi.org/10.1515/biol-2019-0031

[10] Liu, N., Huang, J., Sun, S., Zhou, Z., Zhang, J., Gao, F., Sun, Q. (2015). Expression of matrix metalloproteinase-9, cyclooxygenase-2 and vascular endothelial growth factor are increased in gastrointestinal stromal tumors. International journal of clinical and experimental medicine, 8 (4), 6495-6501.

[11] Inoue, K., A. Fry, E. (2018). Aberrant expression of p16INK4a in human cancers - a new biomarker? Cancer Reports and Reviews, 2 (2). doi: http://doi.org/10.15761/crr.1000145

[12] Laurini, J. A., Blanke, C. D., Cooper, K., Demetri, G. D., Dematteo, R. P., Fletcher, C. D. M. (2019). Protocol for the Examination of Biopsy Specimens From Patients With Gastrointestinal Stromal Tumor (GIST). College of American pathologists. Available at: https://documents.cap.org/protocols/cp-other-gist-biopsy-19-4100.pdf

[13] Fletcher, C. D. M., Berman, J. J., Corless, C., Gorstein, F., Lasota, J., Longley, B. J. et. al. (2002). Diagnosis of gastrointestinal stromal tumors: A consensus approach. Human Pathology, 33 (5), 459-465. doi: http://doi.org/10.1053/hupa.2002.123545

[14] Mei, L., Smith, S. C., Faber, A. C., Trent, J., Grossman, S. R., Stratakis, C. A., Boikos, S. A. (2018). Gastrointestinal Stromal Tumors: The GIST of Precision Medicine. Trends in Cancer, 4 (1), 74-91. doi: http://doi.org/10.1016/j.trecan.2017.11.006

[15] Agaimy, A., Wünsch, P. H. (2007). Distribution of neural cell adhesion molecule (NCAM/CD56) in gastrointestinal stromal tumours and their intra-abdominal mesenchymal mimics. Journal of Clinical Pathology, 61 (4), 499-503. doi: http://doi.org/ 10.1136/jcp.2007.052696

[16] Miettinen, M., Wang, Z.-F., Lasota, J. (2009). DOG1 Antibody in the Differential Diagnosis of Gastrointestinal Stromal Tumors. American Journal of Surgical Pathology, 33(9), 1401-1408. doi: http://doi.org/10.1097/pas.0b013e3181a90ela

[17] Kiśluk, J., Zińczuk, J., Kemona, A., Guzińska-Ustymowicz, K., Żurawska, J., Kędra, B. (2016). Expression of CD117, DOG-1, and IGF-1R in gastrointestinal stromal tumours - an analysis of 70 cases from 2004 to 2010. Gastroenterology Review, 2, 115-122. doi: http://doi.org/10.5114/pg.2015.52587

[18] Swalchick, W., Shamekh, R., Bui, M. M. (2015). Is DOG1 Immunoreactivity Specific to Gastrointestinal Stromal Tumor? Cancer Control, 22 (4), 498-504. doi: http://doi.org/10.1177/107327481502200416

[19] Meena, R. K., Koshti, A., R.K., N., Malik, R., Junwal, A. (2020). Expression of Vimentin, Desmin, CD34 and S100 in Malignant Soft Tissue Tumours in a Study Conducted in Central India. Journal of Evolution of Medical and Dental Sciences, 9 (52), 3977-3981. doi: http://doi.org/10.14260/jemds/2020/869

[20] Haller, F., Agaimy, A., Cameron, S., Beyer, M., Gunawan, B., Happel, N. et. al. (2010). Expression of p16INK4Ain gastrointestinal stromal tumours (GISTs): two different forms exist that independently correlate with poor prognosis. Histopathology, 56 (3), 305-318. doi: http://doi.org/10.1111/j.1365-2559.2010.03478.x

[21] Mendaza, S., Fernández-Irigoyen, J., Santamaría, E., Zudaire, T., Guarch, R., Guerrero-Setas, D. et. al. (2020). Absence of Nuclear p16 Is a Diagnostic and Independent Prognostic Biomarker in Squamous Cell Carcinoma of the Cervix. International Journal of Molecular Sciences, 21 (6), 2125. doi: http://doi.org/10.3390/ijms21062125

How to cite: Miroshnichenko, Y. (2021). Immunohistochemical features of gastrointestinal stromal tumors and their role for differential diagnosis and prognosis. EUREKA: Health Sciences, 6, 10-16. doi: http://doi.org/10.21303/2504-5679.2021.002183 